\title{
For the comments on DNA replication, telomere function and cancer action
}

\author{
Adnan Y. Rojeab* \\ Electrical and Electronic Engineering Department, The London College, UCK, London, UK
}

This is a commentary showing, generally, the aspects of electrical and magnetic actions, which are concerning specifically to the processes of DNA function, as that for those of the replication activity and telomere function, also, for that of the cancer occurrence: For the living kind body's systems, activities and cooperation are, mainly, performed by the form of the electromagnetic signals, where the electrons are playing the main role. Electrical and magnetic signals are being sent and received by the brain [or other control central], to other parts of the body. While, enzymes, proteins, hormones and other biological factors are controlled and be active for the all functions of the living kind systems.

By the advance knowledge on electromagnetic phenomenon and biological actions of the living kind, the functions of genetics and genomics are applied on cloning process, which can be shown briefly as:

Cloning is mainly processed by the effect of electromagnetic action. It is the use of a tiny magnetic field, from an electrical current, to be applied on a cell of a tissue in the body. The magnetic field, then, operates as an anti - dormant factor on the genes of the chromosomes in the cell. It is to let all the genes, of the cell, to be active instead of only, usually, some certain genes, which are belong to that certain tissue of the body. The magnetic action, on the cell, was as if the whole cell becoming just fertilized at once. The main idea of electromagnetic application can be applied and to have the action on the process of the DNA replication and telomere shortening. Applicable can be concerned with the research titled "The processes of DNA replication and the shortening of the telomere are influenced by the action of the magnetic field". It can be show that, the process is mainly cooperated with the electrical and magnetism action, which is acting continuously on human and other kind living.

Magnetism is playing an important main role in DNA replication, (in the nucleus), which is according to the phenomena of both induction and residual magnetisation. These are acting on the surrounding amino acid medium by each DNA strand, as the strand is being a source of magnetic field, to create a new strand. The explanation process of the DNA replication could lead to better understanding of the power activity, ability and the method that how DNA is, usually, in operation. Where this is according to its structure and formation. Therefore, it could be found some way for an operating method to deal with the magnetic action in order to improve the cooperation with DNA activities. In this manner, clinical application, with the Lab application, is giving a great possibility to improve important functions such as that of applying the magnetic field.

Accordingly, the fundamental understanding to the process of DNA replication will lead to possible improvement to the performance of the
DNA from the stage of original cell fertilization. Also, for the process of shortening of the telomere, this is an important and fundamental matter, as it is accounted to be a natural result to be happened for the living kind being. Its performance is according to the phenomenon that been shown in remanant, [residual], magnetisation, which is acting during the replication process. Hence, there is a suggestion as that: it is probably possible to perform this process by the use of the action of the magnetic field [as an action of magnetisation], in order to change the number of base pairs, [which is indicated the size of the telomere length]. Where, as it is shown, this action is, usually, applied during the process of the daughter strand creation, [by the DNA replication].

The action of the magnetic field is, mainly, accounted as a sort of similarity to that of cloning operation process. This can be explained as it is in accordance with results of the DNA replication, which is showing a shortening of the telomere. Then, accordingly, to concern with the result of the telomere function, it is shown that DNA replication is leaving the somatic cells to have shortening their telomeres. This result is different for the cancer cells, as their telomere is lengthening, by the action of enzyme telomerase. Hence, it is believed that, a right application, in this case, is to perform the process of the cancer cells, by the action of magnetism, [or electromagnetic performance], for the aiming of the possibility to cure cancer cells. Hence, [to have a shortening of the telomere as it is in somatic cells], a deliberate magnetic field has to be applied for the shortening of the telomere of the cancer cells. This process, then, could be accounted as a method of a treatment for the cancer cells to, possibly, behave as those of ordinary somatic cells.

When there will be a fully understanding to the behaviour of the DNA replication, and to the causing of the exact origin of the cancer and the function of its mechanism, then, all of this may lead to advance stages to cure the cancer.

The previous meaning, I believe, is included in the paper on cancer, titled "Novel strategy of a method to cure cancer". it is suggested that, the cancer is a mechanism trying to eliminate the sever danger actions, which are working against the vital genes in the cells. Accordingly, the cancer is not, accounted, as a danger by itself. The dangerous to the kind living is by some factors, and by those of operating traditional methods, in the clinics, which are used in trying to cure cancer. Also,

Correspondence to: Adnan Y. Rojeab, Electrical and Electronic Engineering Department, The London College, UCK, London, UK; E-mail: rojeab@btinternet.com

Received: November 28, 2015; Accepted: December 08, 2015; Published: December 11, 2015 
as it is previously mentioned, one of the suggestions for the treatment methods to cure cancer is to apply a certain amount and direction of a magnetic field in order to reduce the number of base pares, which been added to the telomere by the enzyme telomerase, during DNA replication.

The magnetic action is, accounted, as an opposing process to the function of the enzyme telomerase. This is practically means that, as if a certain force is acting on the DNA to be replicated in a similar manner to that of the originally traditional somatic cells.

Now, to deal with the cancer cells, the applied magnetic field should be arranged, [during the DNA replication], for a certain amount and direction, to be possibly acting against the enzyme telomerase. Therefore, it may show a shortening of the telomere instead of lengthening it, [as shortening the telomere is the characteristic of the somatic cell].

In other words, it is, greatly, to apply a suitable method to be able to inhibit the action of the enzyme telomerase, [to be as that action concerned with the somatic cells].

In the other hand, it is, also, true that, lengthening the telomere is entirely important and it is a fundamental process for some type of cells of the body of the kind living. Lengthening the telomere is the natural and original process for generating and existing of the germ cells, [as sperms and ova, in some kind being], and other types of similar function cells, as those of stem cells. The telomere lengthening process of these cells is similar to that of the cancer cells, while germ and stem cells are fundamentally important and out of harming to the human, while the cancer is in opposite.

It is, also, important to emphasize on the main DNA process and telomere functions during DNA replication, in somatic cells:

\section{Magnetic flux action on the DNA and the telomere}

This is to concerned with the improvement to the function of the telomere of the somatic cells. For a vast understanding to the inherent reaction of the DNA and that of somatic cells by the effect of the magnetisation: where, the process is going to apply such a magnetic action method, during DNA replication. The method is to use a certain range of magnitudes and directions of magnetic flux strength, during the replication process.

Hence, the target is, mainly, to discover any DNA telomere change, such as that of the level of activity, molecule deformation, original chemical change or any different shape structure, which is possible to occur. Then, this sort of application will give a broad range of knowledge, for the greater understanding to the nature of DNA functions, including its process of replication.

\section{Telomere shortening and body strength}

It is believed that there is a certain telomere strength characteristic, which is in cooperation with the strength of the cell, and then with that of the whole body. It is a sort of telomere pointing out to the measuring level of the activity and mobility of the body as a whole.

\section{This telomere characteristic may be explained as the following:}

The shortening of the telomere, during DNA replication, is a continuing process associating with that of the frequent of the DNA replication. This means that the continuous shortening of the telomere is greatly dependant on that of the growth of the life of the kind living. Hence, the main relationship is an inversely one between the growth, [which can be accounted as an oldness], of the kind living and that of the reduced, (loosing), the number of the base pairs of the telomere. [length of the telomere]. The shorter the number of the base pairs is the older, [aged], of the kind living. Also, it is shown that (after certain age of the life of the kind living), the older [aged] kind living, is the weaker in the general ability and activity of the body. This may show that, after a certain age: the shorter is of the telomere, it is the inhibiting, [weakening], of the ability and activity of the kind living. This can be shown that, as for human person example, an old and weighted person is less ability and mobile activity than a young person of less, but reasonable, weight, [as the weighting means here that the greater number of cells].

\section{Comment on the critical length of the telomere, number of the base pairs}

The critical number of the base pairs, [telomere length], is causing the DNA to be stopped of its replication process, until the cell dies. It is believed that this occurring is for an order to keep the full secure manner of the complete gene characteristics of the DNA, which is to be for the exactly the same certain type of the kind living. While, if suppose that, the DNA is continued to be replicated after the stage of the critical length of the telomere, then it means that this extra DNA replication will cause a shortening [loosing] in some of the genes, which are positioned near the telomere. Hence, the result of this process will leave the DNA molecule on a missing [loosing] part of its characteristics. This means that the DNA, [and then the cell itself] is, now, belong to another sort of kind living, which is entirely different than that of the original one. Therefore, the mechanism of the dying of the cell, after critical length of the telomere, is of importance to prevent any dangerous or abnormality could be existing.

\section{Comment on the existing of the magnetic field}

It can be recognised that both appearance and that of absent of the magnetic field are very important matter for the replication of the DNA, and then for the cell and then to the kind living. It is concerning with the previous stated for the action of the magnetic field on the replication of the DNA, each strand of the two strands is acting by its own magnetic effect on the surrounding nucleus amino acid, to produce a daughter strands, [of the DNA]. The great importance of this process, is because it is the original and fundamental for the continuation of the exactly the same type of the kind living. Which is to be on conservation of the whole characteristics of the exact kind living, [genes ancestors]. Also, the fact is that, it is only working for a certain time and stage of the living cell function.

While, it is, also, fundamentally important to prevent the action and existence of the magnetic field in the DNA molecule [as a whole entity]. This is because that, if there is a continuation of the exciting of the magnetic effect in the DNA molecule, [as a whole entity], then it will show a raped replication processes of the DNA without stopping. This will lead, obviously, to a situation that the number of chromosomes will be varied and in continuously increasing, in the nucleus, which is showing a change to the original characteristics of the type of the kind living fundamentally.

Then, both performing, which are, acting and non- acting of the magnetic field, at different stages of the DNA functioning are of great important. 
Finally: this commentary is giving a great attention to the action of the electromagnetic phenomena. It is believed to have the control action on many biological functions. DNA replication and telomere functioning and cancer cure, are some examples to be shown for the proven their performances and to have advanced and useful results for kind living, when the improvement is cooperating with the electromagnetic phenomenon.
In each of these processes and steps, it is required the immediate clinical proven and for advanced studies in the order to assess the level of the validating for the points been risen. This is especially for the cancer cure, as it is here, is far from the traditional direction of treatment.

Copyright: $\odot 2015$ Rojeab AY. This is an open-access article distributed under the terms of the Creative Commons Attribution License, which permits unrestricted use, distribution, and reproduction in any medium, provided the original author and source are credited. 